\title{
The impact of local control on overall survival after stereotactic body radiotherapy for liver and lung metastases from colorectal cancer: a combined analysis of 388 patients with 500 metastases
}

Rainer J. Klement ${ }^{1}$ (D, N. Abbasi-Senger ${ }^{2}$, S. Adebahr ${ }^{3}$, H. Alheid ${ }^{4}$, M. Allgaeuer ${ }^{5}$, G. Becker $^{6}$, O. Blanck $^{7}$, J. Boda-Heggemann ${ }^{8}$, T. Brunner ${ }^{3}$, M. Duma ${ }^{9}$, M. J. Eble ${ }^{10}$, I. Ernst ${ }^{11}$, S. Gerum ${ }^{12}$, D. Habermehl ${ }^{9,13}$, P. Hass ${ }^{14}$, C. Henkenberens ${ }^{15}$, G. Hildebrandt ${ }^{16}$, D. Imhoff ${ }^{17}$, H. Kahl ${ }^{18}$, N. D. Klass ${ }^{19}$, R. Krempien ${ }^{20}$, V. Lewitzki ${ }^{21}$, F. Lohaus $^{22}$, C. Ostheimer ${ }^{23}$, A. Papachristofilou ${ }^{24}$, C. Petersen ${ }^{25}$, J. Rieber ${ }^{13}$, T. Schneider ${ }^{26}$, E. Schrade ${ }^{27}$, R. Semrau ${ }^{28}$, S. Wachter ${ }^{29}$, A. Wittig ${ }^{2,30}$, M. Guckenberger ${ }^{31}$ and N. Andratschke ${ }^{31^{*}}$ (D)

\begin{abstract}
Background: The aim of this analysis was to model the effect of local control (LC) on overall survival (OS) in patients treated with stereotactic body radiotherapy (SBRT) for liver or lung metastases from colorectal cancer.

Methods: The analysis is based on pooled data from two retrospective SBRT databases for pulmonary and hepatic metastases from 27 centers from Germany and Switzerland. Only patients with metastases from colorectal cancer were considered to avoid histology as a confounding factor. An illness-death model was employed to model the relationship between LC and OS.

Results: Three hundred eighty-eight patients with 500 metastatic lesions (lung $n=209$, liver $n=291$ ) were included and analyzed. Median follow-up time for local recurrence assessment was 12.1 months. Ninety-nine patients with 112 lesions experienced local failure. Seventy-one of these patients died after local failure. Median survival time was 27.9 months in all patients and 25.4 months versus 30.6 months in patients with and without local failure after SBRT. The baseline risk of death after local failure exceeds the baseline risk of death without local failure at 10 months indicating better survival with LC.
\end{abstract}

Conclusion: In CRC patients with lung or liver metastases, our findings suggest improved long-term OS by achieving metastatic disease control using SBRT in patients with a projected OS estimate of $>12$ months.

Keywords: Colorectal cancer, IIIness-death model, Liver metastases, Lung metastases, Tumor control probability, Stereotactic body radiation therapy

\footnotetext{
* Correspondence: Nicolaus.Andratschke@usz.ch

${ }^{31}$ Department of Radiation Oncology, University Hospital Zurich, University of

Zurich, Rämistrasse 100, 8091 Zurich, Switzerland

Full list of author information is available at the end of the article
}

(c) The Author(s). 2019 Open Access This article is distributed under the terms of the Creative Commons Attribution 4.0 International License (http://creativecommons.org/licenses/by/4.0/), which permits unrestricted use, distribution, and reproduction in any medium, provided you give appropriate credit to the original author(s) and the source, provide a link to the Creative Commons license, and indicate if changes were made. The Creative Commons Public Domain Dedication waiver (http://creativecommons.org/publicdomain/zero/1.0/) applies to the data made available in this article, unless otherwise stated. 


\section{Background}

Colorectal cancer (CRC) is the third most common malignancy with a global burden expected to increase to 2.2 million new cases and 1.1 million deaths by 2030 [1]. Liver is the most common site of CRC metastasis and patients with liver metastases have been found to have a particularly poor prognosis with significantly reduced overall survival (OS) [2]. Still, in selected CRC patients with limited liver or lung metastases longer-term survival can be achieved with complete surgical resection of all metastatic lesions reaching 5-year OS rates up to $40 \%$ $[3,4]$. Due to technological innovations and based on positive experiences with treating primary non-small cell lung cancer (NSCLC) patients, stereotactic body radiotherapy (SBRT) is increasingly adopted to treat pulmonary and hepatic metastases. So far, however, dose prescriptions have been mostly based on the experiences made with primary NSCLC in case of pulmonary metastases or on maximally tolerable doses for organs at risk in case of liver irradiation. Prospective studies investigating optimal dosing schedules are lacking for SBRT of extra-cranial metastases in general. For CRC metastases in particular, some have even claimed that the site of metastatic growth has an influence on radiosensitivity and thus local control (LC) probability [5]. To our knowledge, however, this has not yet been studied using sophisticated models of patient outcomes since no sufficient data has been collected so far.

An even more pressing question in this context is what value optimizing the probability of LC would have for longer-term OS. The rationale for using SBRT to treat extra-cranial metastases was partly based on the observation that patients with a limited number of pulmonary, hepatic or brain metastasis experienced survival benefits after complete surgical resection [6]. Hints for similar benefits of extra-cranial SBRT have so far been obtained in single-institutional studies of a small number of subjects [7]. Recently, a multicenter randomized phase II study for oligometastasized NSCLC could demonstrate a significantly improved progression-free survival with local treatment in patients responding to first-line chemotherapy versus maintenance chemotherapy alone [8]. The recently presented outcome data of the EORTC-NCRI CCSG-ALM Intergroup 40,004 trial could demonstrate for the first time a positive effect of a local ablative therapy in the form of radiofrequency ablation on OS in patients with liver metastases from CRC in addition to chemotherapy [9].

Sophisticated modeling of the putative importance of achieving LC for OS has not yet been performed, but may - with the scarce prospective data available - aid in the decision making on local treatment in oligometastasized patients. To address this highly relevant issue we have compiled the largest sample of colorectal lung and liver metastases treated with SBRT from two separate databases of the SBRT working group of the German Society for Radiation Oncology (DEGRO). This will be used to model the outcome after SBRT with regard to LC as well as OS with an emphasis on the role that the site of metastasis might play.

\section{Methods \\ Data preparation}

The analysis is based on pooled data from two large retrospective databases of SBRT treatments for pulmonary and hepatic metastases compiled from a total of 27 German and Swiss hospitals, all members of the DEGRO SBRT working group. All treatments performed on colorectal cancer patients were pooled into a new database, totaling 538 individual metastases treated with SBRT. Detailed descriptions of the separate databases have already been published [10, 11]. The multicenter data collection and analysis was approved by the respective local Ethics committees of the principle investigator's institutions. For the current study, we excluded 38 metastases with no post-treatment evaluation of LC or OS, resulting in 500 metastases belonging to 388 patients. All clinical and treatment related variables relevant for the current analysis are compiled in Table 1.

Local recurrence for the SBRT treated lesions was defined as either reappearance after complete remission or re-growth after initial partial response to SBRT in followup CT or MRI scans. PET-CT scans were used by some centers in equivocal cases to confirm local recurrence. In case that death and local failure were recorded at the same date (this was the case for only 2 metastases), we adopted the convention that death happened first and treated LC as censored [12]. For modeling, all prescriptions were converted to biologically effective doses at the isocenter defined as $\mathrm{BED}_{\text {iso }}=n d\left(1+\frac{d}{\alpha / \beta}\right)$ where $n$ is the number of fractions, $d$ the fraction dose at the isocenter and $\alpha / \beta$ assumed as $10 \mathrm{~Gy}$.

The binary variable "chemotherapy prior to treatment" was set to 1 if a patient had received chemotherapy prior to SBRT at least once, and maximum tumor volume did correspond to the largest metastasis treated. Table 1 provides details on all covariates and outcomes considered for modeling.

Two hundred fifty-five patients were treated for a total of 291 liver and 133 patients for a total of 209 lung metastases. In the dichotomization of the dose calculation algorithm, "Advanced" refers to either Collapsed Cone, Analytical Anisotropic Algorithm or Monte Carlo based algorithms.

\section{Model description}

Our main interest lies in the relationship between LC and OS. We model these two outcomes within the 
Table 1 Variables and outcomes in our sample of 500 CRC metastases

\begin{tabular}{|c|c|c|c|c|c|}
\hline Covariates and outcomes & $\mathrm{n}$ & Value & Liver & Lung & $p$-value \\
\hline Sex & 388 & & & & \\
\hline Male & 271 & 69.8 & 72.2 & 65.4 & 0.200 \\
\hline Female & 117 & 30.2 & 27.8 & 34.6 & \\
\hline Age [years] & 388 & 66 (24-93) & $66(24-93)$ & $70(38-36)$ & 0.00399 \\
\hline Baseline Karnofsky index & 283 & & & & \\
\hline$<90$ & 97 & 34.2 & 30.6 & 40.9 & 0.125 \\
\hline$\geq 90$ & 186 & 65.7 & 69.4 & 59.1 & \\
\hline Solitary metastasis & 304 & & & & \\
\hline Yes & 110 & 36.2 & 33.2 & 40.7 & 0.184 \\
\hline No & 194 & 63.8 & 66.8 & 59.3 & \\
\hline Number of treated metastases & 388 & & & & \\
\hline 1 & 321 & 82.7 & 89.4 & 69.9 & \\
\hline 2 & 42 & 10.8 & 8.2 & 15.8 & \\
\hline 3 & 13 & 3.4 & 1.6 & 6.8 & \\
\hline 4 & 7 & 1.8 & 0.4 & 4.5 & \\
\hline 5 & 2 & 0.5 & 0.4 & 0.7 & \\
\hline 6 & 3 & 0.8 & 0 & 2.3 & \\
\hline Tumor site & 500 & & & & \\
\hline Liver & 291 & 58.2 & & & \\
\hline Lung & 209 & 41.8 & & & \\
\hline Tumor volume [ccm] (gross tumor volume, GTV) & 342 & $9.20(0.07-699)$ & $26.0(0.8-699)$ & $3.1(0.07-268)$ & $<0.0001$ \\
\hline Chemotherapy prior to SBRT & 430 & & & & \\
\hline Yes & 332 & 77.2 & 84.5 & 68.5 & $<0.0001$ \\
\hline No & 98 & 22.8 & 15.5 & 31.5 & \\
\hline Dose calculation algorithm & 496 & & & & \\
\hline Pencil beam & 199 & 40.1 & 56.4 & 17.7 & $<0.0001$ \\
\hline Advanced & 297 & 59.9 & 43.6 & 82.3 & \\
\hline Motion management & 500 & & & & \\
\hline Free breathing & 347 & 69.4 & 75.3 & 73.7 & 0.755 \\
\hline Advanced & 153 & 30.6 & 24.7 & 26.3 & \\
\hline $\mathrm{BED}_{\text {iso }}\left[\mathrm{Gy}_{10}\right]$ & 500 & $126.9(37.5-309.4)$ & $124.8(37.5-234.5)$ & $141.1(39.4-309.4)$ & $<0.0001$ \\
\hline Outcomes & 388 & & & & \\
\hline Local failure and censored prior to death & 28 & 7.2 & 7.5 & 6.8 & $<0.0001$ \\
\hline Local failure and death & 71 & 18.3 & 24.3 & 6.8 & \\
\hline Death without local failure & 133 & 34.3 & 36.5 & 30.0 & \\
\hline Censored prior to local failure or death & 156 & 40.2 & 31.8 & 56.4 & \\
\hline
\end{tabular}

Values for continuous variables are given as median (range), those for categorical variables as frequencies in percent. The $\mathrm{p}$-values refer to testing for differences between liver and lung metastases with respect to the variable specified in each row. The Wilcoxon rank sum test and Fisher's exact test were used to compare continuous and categorical variables, respectively. The variable "solitary metastasis" was coded as "yes" if only lung or liver was involved by a singular metastasis. Beyond this information, the exact location and number of additional metastases has not been encoded

context of a multistate model, or more specific, a so-called illness-death model [13] where death can occur after local failure but not vice versa. In the illness-death model there are three possible transitions (Fig. 1): (1) treatment to relapse; (2) treatment to death; (3) relapse to death.
We assumed proportional cause-specific hazards and included frailty terms to account for possible heterogeneity between hospitals. For transition (1) the unit of interest is the treated lesion. Clustering of metastases within patients was neglected due to the very small number of metastases associated with each patient. For 


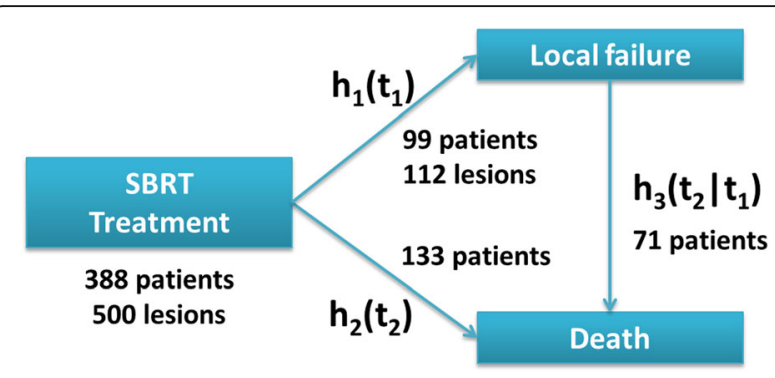

Fig. 1 Conception of the illness-death modeling framework applied to the study of local failure and death in metastatic rectal cancer patients treated with SBRT. Starting from the state "SBRT treatment", patients can either transition into the state "Local failure" (the nonterminal event occuring at time $T_{1}$ ) or "Death" (the terminal event occurring at time $T_{2}$ ). A third transition from "Local failure" to "Death" is also possible, but not vice versa. The rates at which patients transition from one state to the other are specified by three corresponding hazard functions that we model using Eqs. (1-3). $h_{1}\left(t_{1}\right)$ is the hazard rate for local failure from SBRT at a given point in time $t_{1}$, given that neither local failure or death have occurred before $t_{1} . h_{2}\left(t_{2}\right)$ is the hazard rate for death after SBRT at a given point in time $t_{2}$, given that neither local failure nor death have occurred before $t_{2}$. Finally, $h_{3}\left(t_{2} \mid t_{1}\right)$ is the hazard rate of death at a given time point $t_{2}$ given that local failure has been observed at $T_{1}$ $=t_{1}$ and that death has not occurred before $t_{2}$

transitions (2) and (3) the unit of interest was the patient. For all three transitions the clustering of patients within hospitals was accounted for using a shared frailty model [14]. In case of multiple treatments we define the first treatment as the time origin and time of the first relapse as the failure time.

Let $T_{i j k 1}$ denote the time from treatment until relapse for metastasis $k$ belonging to patient $j$ from hospital $i$ with $k=1, \ldots, n_{i j}, j=1, \ldots, s_{i}$ and $i=1, \ldots, h$. Furthermore, let $T_{i j 2}$ denote the time from first treatment until death for patient $j$. The three possible transitions are then specified by the three cause-specific hazard functions:

$$
h_{1}\left(t_{i j k 1} \mid w_{i 1}, \boldsymbol{X}_{i j k 1}\right)=h_{01}\left(t_{i j k 1}\right) \exp \left(\boldsymbol{X}_{i j k 1}^{T} \boldsymbol{\beta}_{1}+w_{i 1}\right), t_{i j k 1}>0
$$

$$
h_{2}\left(t_{i j 2} \mid w_{i 2}, \boldsymbol{X}_{i j 2}\right)=h_{02}\left(t_{i j 2}\right) \exp \left(\boldsymbol{X}_{i j 2}^{T} \boldsymbol{\beta}_{2}+w_{i 2}\right), t_{i j 2}>0
$$

$$
\begin{gathered}
h_{3}\left(t_{i j 2} \mid\left\{t_{i j k 1}\right\}, w_{i 3}, \boldsymbol{X}_{i j 3}\right)=h_{03}\left(t_{i j 2} \mid\left\{t_{i j k 1}\right\}\right) \\
\exp \left(\boldsymbol{X}_{i j 3}^{T} \boldsymbol{\beta}_{3}+w_{i 3}\right), \\
t_{i j 2}>t_{i j k 1}
\end{gathered}
$$

Here $\boldsymbol{w}_{i}=\left(w_{i 1}, w_{i 2}, w_{i 3}\right)$ is a vector of hospital-specific random effects (each belonging to one of the three transitions) and $\boldsymbol{\beta}_{g}, g \in\{1,2,3\}$ denotes the transition-specific fixed effects. The random effects $\boldsymbol{w}_{i}$ were assumed to stem from a Gaussian distribution with mean 0 and variance $\sigma_{i}^{2}$.

The hazard $h_{3}$ in Eq. (3) defines the rate of death of patient $j$ from hospital $i$ following the occurrence of local failure at time $T_{i j k 1}=t_{i j k 1}$. This hazard principally depends on the set of failure times $\left\{t_{i j k 1}\right\}_{k=1, \ldots, n_{i j}}$ of the $n_{i j}$ metastases of patient $j$. To simplify we apply the Markov assumption, $h_{3}\left(t_{i j 2} \mid\left\{t_{i j k 1}\right\}\right)=h_{3}\left(t_{i j 2}\right)$, in which the hazard of death does not depend on the particular time of tumor recurrence. This can be interpreted as stating that a patient's risk of death at any time after the first SBRT is initially described by the hazard in Eq. (2), but if and when a local relapse has occurred takes on the form of Eq. (3) [13]. The hazard $h_{3}$, which is estimated using patients that have experienced local relapse, is theoretically defined for every patient beginning from the earliest time point that a local relapse has been recorded in our data (which was 2 months). The Markov assumption is useful for inferring the general importance of achieving LC for a patient's probability of OS. In this case, the hazards $h_{2}\left(t_{i j 2}\right)$ and $h_{3}\left(t_{i j 2}\right)$ correspond to the hazards of death at time $t_{i j 2}$ given that death has not occurred before $t_{i j 2}$ and all metastases have been controlled or not before $t_{i j 2}$, respectively. The so-called explanatory hazard ratio $h_{3} / h_{2}$ characterizes the dependence between $T_{i j-}$ ${ }_{k 1}$ and $T_{i j 2}$. If $h_{3} / h_{2}=1$, the occurrence of $T_{i j k 1}$ has no effect on the hazard of dying at $T_{i j 2}$, while when $h_{3} / h_{2}>1$ at $t_{i j 2}$ this indicates that for a fixed value of the frailty and covariates, the risk of death is higher if a relapse had occurred before $t_{i j 2}$ [15].

Alternatively, one could think of treating local failure as a time-dependent covariate $Z_{j}(t)$ which takes on value 0 as long as LC is achieved, but jumps to 1 if local failure occurs in patient $j$. In this case, the hazard of death would be $h\left(t_{i j} \mid w_{i}, \boldsymbol{X}_{i j}\right)=h_{0}\left(t_{i j}\right) \exp \left(Z_{j}(t) \beta_{0}+\boldsymbol{X}_{i j}^{T} \beta+w_{i}\right)$ so that $h_{2}$ and $h_{3}$ would have the same baseline hazard, their ratio would be given as $h_{3} / h_{2}=\exp \left(\beta_{0}\right)$, and $h_{1}$ would be left unspecified. These restrictions do not apply to the illness-death model whose structure naturally accounts for the relation between LC and OS.

Models were fit using maximum integrated partial likelihood estimation by the Laplace approximation with the $\mathrm{R}$ package coxme. The refine.n option was used to confirm the goodness of the Laplace approximation via Monte Carlo control sampling.

Given a specification of the baseline hazard function, the probability of local failure or death between time $t$ and horizon time $t+w$ can be estimated. We therefore complemented the analysis by approximating the baseline hazard function on the basis of cubic M-splines that were fit using the frailtypack package [16]. A lognormal frailty was used which is equivalent to Gaussian random effects [14]. The frailtypack routine uses maximum 
penalized likelihood estimation based on the robust Marquardt algorithm [16]. For each transition, the number of knots for the splines was set to 9 and the optimal smoothing parameter $\mathrm{K}$ in the penalized log likelihood was estimated by cross-validation [16].

\section{Variable selection and model preparation}

For survival regression, it is generally recommended to restrict the number of covariates to approximately the number of events divided by 15 [17]. We tried to use this as a constraint to select an appropriate number of covariates for each of the three transitions (Eqs. 1-3) from the full set shown in Table 1 based on clinical interest and knowledge from previous modeling studies.

Chemotherapy prior to SBRT treatment (missing for $14 \%$ of metastases) and tumor volume (missing for 31.6\%) were judged as putative prognostic factors for LC, and baseline Karnofsky performance score (KPS, missing for $27 \%$ of patients) as well as presence of multiple metastases (missing for $21.6 \%$ ) as possible prognostic factors for OS. We imputation by chained equations (mice) [18] to impute the missing values for these covariates in order to maximize the number of cases for modeling. The ideal set of predictors for each variable was determined with help of the mice package function quickpred by requiring a minimal correlation of 0.2 and minimum proportion of usable cases of 0.25 . For 99 of the 158 lesions with unknown tumor volume, the PTV volume was available and used in the imputation. Sensitivity to the particular imputation of the variables was checked by performing a total of $50 \mathrm{im}$ putations, each time refitting the models and pooling the results together using the pool function from the mice package in $\mathrm{R}$ [18].

All covariates except $\mathrm{BED}_{\text {iso }}$ and tumor volume were categorized to $0 / 1, \mathrm{BED}_{\text {iso }}$ and tumor volume were standardized by subtracting the mean and dividing by two standard deviations [19].

\section{Statistical tests}

Fisher's exact test and the Wilcoxon rank sum test were used to compare categorical and continuous variables, respectively, between liver and lung metastases. Two-sided Wald tests were used to obtain $p$-values for regression coefficients.

\section{Results}

In total, 388 patients with 500 metastatic lesions (lung $n$ $=209$, liver $n=291$ ) were included and analyzed. Most frequent dose prescriptions were $5 \times 7$ Gy @65\% isodose line (11.6\% of metastases), $1 \times 24 \mathrm{~Gy} @ 80 \%$ (7.4\%) and $3 \times 12.5$ Gy @65\% (5.4\%). Chemotherapy was significantly more often administered prior to SBRT of liver (84.5\%) compared to lung (68.5\%) metastases (Table 1).
Also, lung metastases received significantly higher $\mathrm{BED}_{\text {iso }}$ than liver metastases. Tumor volumes were significantly larger in liver compared to lung metastases, and advanced dose calculation algorithms were used significantly more often for planning treatment of the latter. Due to these differences the site of tumor location was included as an important confounding factor into each of the following models.

Median follow-up time for LC was 12.1 months (range $0.03-95.7)$ and 17.8 months $(0.16-151.8)$ for OS. There were 3 metastases in 2 patients that were lost to LC assessment directly after the end of SBRT treatment which explains the short FU duration of 0.03 months; both patients died 21 and 53 days after beginning of SBRT, respectively. In total, 99 patients with 112 lesions experienced local failure. Seventy-one of these patients have died after experiencing local failure. Median survival time was 27.9 months $(95 \%$ CI 24.4-31.8) in all patients and 25.4 months (95\% CI 23.6-33.0) versus 30.6 months (95\% CI 24.5-37.4) in patients with and without local failure after SBRT $(p=0.19)$, respectively. Local relapses were recorded in $31.3 \%$ of liver metastases compared to $10.0 \%$ of lung metastases $(p<0.0001)$.

Table 2 provides an overview of all covariates selected for modeling each transition together with their regression coefficients. In the multivariable analyses, smaller tumor volumes, advanced motion management and dose calculation techniques were non-significantly associated with higher LC rates; significantly better tumor control was found for lung metastasis compared to liver metastases, no prior chemotherapy and higher $\mathrm{BED}_{\text {iso. }}$.

Figure 2 shows the Kaplan-Meier tumor control probability (TCP) curves for both liver and lung metastases together with the predictions of the lognormal frailty model for an average treatment. In this context, "average" refers to the mean and most frequent value for continuous and categorical variables, respectively, of lung and liver metastases. The model predicts that for a $90 \%$ TCP at 2 years, BEDiso of $99 \mathrm{~Gy}_{10}$ (lung) and $187 \mathrm{~Gy}_{10}$ (liver) would be sufficient with no prior chemotherapy, but $211 \mathrm{~Gy}_{10}$ (lung) and $300 \mathrm{~Gy}_{10}$ (liver) would be needed if prior chemotherapy would have been given (Table 3).

For patients without local recurrence, baseline KPS and maximum tumor diameter were found to be significant for OS. If and when local failure occurred, maximum tumor diameter remained highly associated with worse OS, while there was a weaker, yet significant association for chemotherapy prior to SBRT to improve OS (Table 2). We found that because of the significantly larger sizes of liver metastases, controlling for maximum tumor diameter was important for diminishing an otherwise significant effect of liver metastases predicting worse OS in transition 2. 
Table 2 Covariates selected for modeling each transition (Eqs. 1-3) and their estimated regression coefficients expressed as hazard ratios

\begin{tabular}{|c|c|c|c|c|c|c|c|c|c|}
\hline \multirow{2}{*}{$\begin{array}{l}\text { Transition } \\
\text { Covariates }\end{array}$} & \multicolumn{3}{|c|}{ Treatment to local failure (1) } & \multicolumn{3}{|c|}{ Treatment to death (2) } & \multicolumn{3}{|c|}{ Local failure to Death (3) } \\
\hline & $\exp (\beta)$ & $95 \% \mathrm{Cl}$ & $p$-value & $\exp (\beta)$ & $95 \% \mathrm{Cl}$ & $p$-value & $\exp (\beta)$ & $95 \% \mathrm{Cl}$ & $p$-value \\
\hline Sex: Female & & & & 0.97 & $0.65-1.44$ & 0.867 & & & \\
\hline Age $\geq 66$ & & & & 1.13 & $0.75-1.69$ & 0.564 & & & \\
\hline$K P S \geq 90$ & & & & 0.47 & $0.29-0.78$ & 0.0037 & 1.30 & $0.56-3.02$ & 0.534 \\
\hline Tumor site: Lung & 0.42 & $0.25-0.70$ & 0.0010 & 0.89 & $0.56-1.41$ & 0.611 & 1.30 & $0.56-3.05$ & 0.537 \\
\hline Solitary metastasis: Yes & & & & 0.83 & $0.53-1.29$ & 0.405 & 0.55 & $0.23-1.30$ & 0.174 \\
\hline Number of treated metastases $>1$ & & & & 0.96 & $0.59-1.56$ & 0.861 & & & \\
\hline Chemotherapy prior to SBRT: Yes & 3.64 & $1.58-8.36$ & 0.0024 & 1.19 & $0.71-1.98$ & 0.508 & 0.19 & $0.04-0.84$ & 0.028 \\
\hline Tumor volume & 1.20 & $0.89-1.63$ & 0.232 & 1.99 & $1.47-2.69$ & $<0.0001$ & 2.12 & $1.25-3.58$ & 0.0053 \\
\hline Motion management: Advanced & 0.81 & $0.49-1.34$ & 0.411 & & & & & & \\
\hline Dose calculation: Advanced & 0.86 & $0.55-1.34$ & 0.497 & & & & & & \\
\hline $\mathrm{BED}_{\text {iso }}$ & 0.39 & $0.25-0.64$ & 0.00013 & & & & & & \\
\hline
\end{tabular}

If a variable was not used as a covariate for modeling the hazard of a particular transition, its corresponding cell has been left empty. For Transition (2), tumor volume refers to the maximum tumor volume of all treated metastases within a particular patient

KPS Baseline Karnofsky performance status

Figure 3 shows the baseline hazard ratio $\left(h_{03} / h_{02}\right)$ between death after local recurrence (transition 3) and death without local recurrence (transition 2) as a function of time starting at 2 months, the earliest time of recurrence recorded in our database. The baseline hazards correspond to the hazard of death when all fixed and random effects are zero. It can be clearly seen that from about 10 months on, the baseline risk of death after local failure significantly exceeds the baseline risk of death without local failure, and the ratio increases with time indicating better survival after LC is achieved. This implies that if the overall prognosis is judged to be beyond 1 year LC is a decisive factor for OS.

Figure 4 shows the predicted cumulative probability of dying without (transition 2) or after (transition 3) experiencing local recurrence. Two predictions for an average patient with either lung metastasis (right panel) or liver metastasis (left panel) are shown. The cumulative death

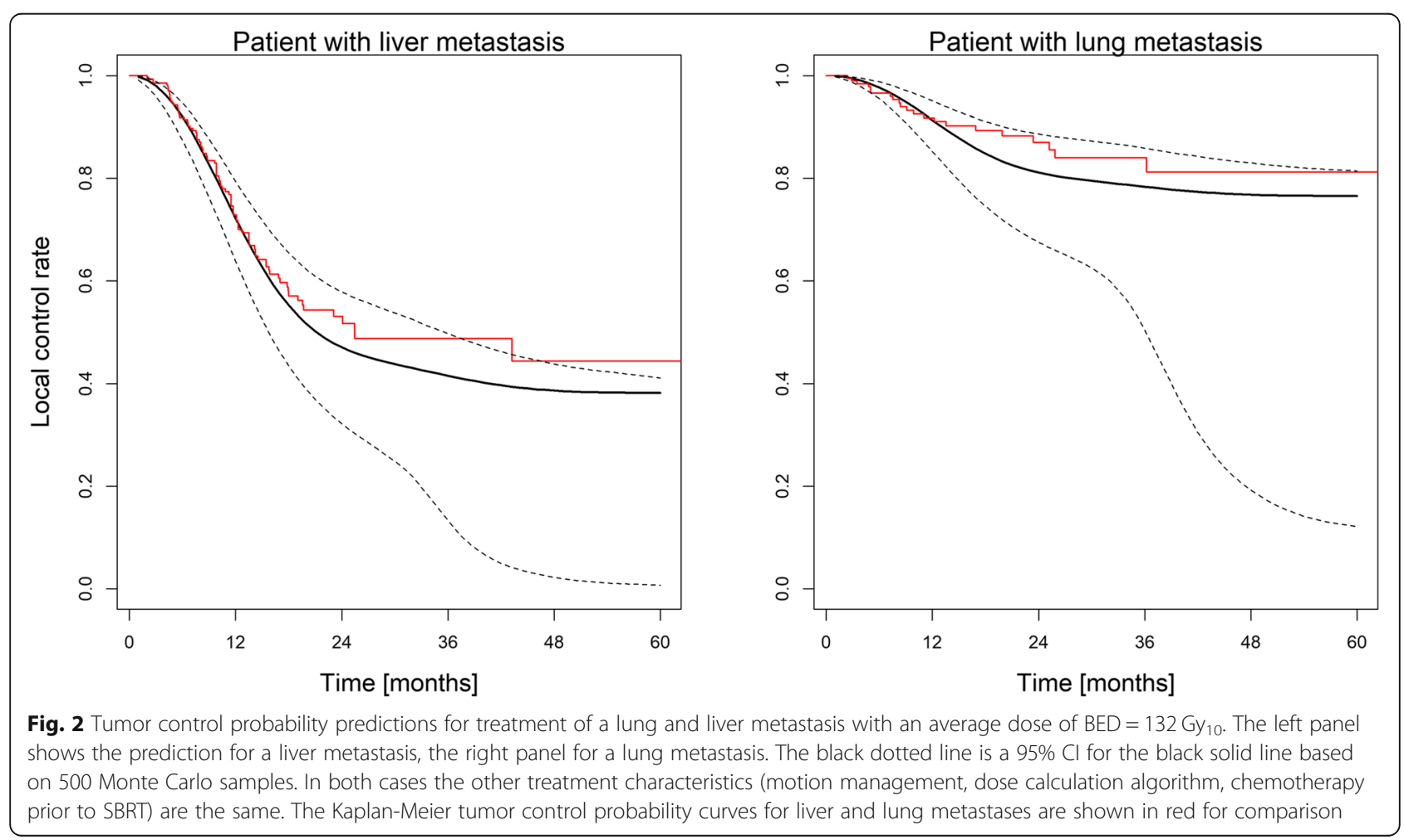


Table $3 \mathrm{BED}_{\text {iso }}$ converted to clinically applicable dose fractionation schedules to achieve at least $90 \%$ local control at 2 years of CRC metastases

\begin{tabular}{|c|c|c|c|c|}
\hline Tumor location & No prior Chemo & & Prior Chemo & \\
\hline \multirow[t]{2}{*}{ Lung } & $99 \pm 15 \mathrm{~Gy}_{10} \mathrm{BED}_{\text {iso }}$ & 3×9Gy@65\% & $211 \pm 19 \mathrm{~Gy}_{10} \mathrm{BED}_{\text {iso }}$ & 3×15Gy@65\% \\
\hline & & 8×5 Gy @ 65\% & & $5 \times 10.5$ Gy @ 65\% \\
\hline \multirow[t]{2}{*}{ Liver } & $187 \pm 19 \mathrm{~Gy}_{10} \mathrm{BED}_{\text {iso }}$ & 3×14 Gy @ 65\% & $300 \pm 39 \mathrm{~Gy}_{10} \mathrm{BED}_{\text {iso }}$ & 3×18Gy@65\% \\
\hline & & 5×10Gy @ 65\% & & 5×13Gy@65\% \\
\hline
\end{tabular}

probability for dying after local recurrence is almost equal for lung and liver metastases, with slightly differing $95 \%$ confidence bands indicating a similar effect of local recurrence on OS. In contrast, the cumulative probability of dying without experiencing local recurrence is higher in patients with liver than in patients with lung metastases indicating a generally worse prognosis of patients with liver compared to lung metastases. Still, in both cases the probability of making the transition 3 (death after local recurrence) quickly exceeds that for transition 2 (death without local recurrence), consistent with the behavior of the baseline hazard ratio shown in Fig. 3, underlining the impact of LC on OS.

\section{Discussion}

Although Hellman and Weichselbaum coined the concept of oligometastases as a distinct state with its own biology in 1995 [20], it only recently started to be more thoroughly investigated in the context of SBRT [7, 21]. Nevertheless, up until recently, there was no clinical proof that oligometastatic patients really do benefit from local interventions such as surgery or SBRT. A possible hint for a benefit could be derived from surgical series indicating worse OS after microscopic incomplete resection compared to complete resection, indicating the need for LC of the respective metastases [22]. With the outcome of the EORTC-NCRI CCSG-ALM Intergroup 40,004 trial, the first prospective data showing a positive effect of a local ablative treatment on OS - in this case radiofrequency ablation - in patients with liver metastases from CRC became available [9].

On the other hand, SBRT series on oligometastatic patients included most frequently a mixture of histologies rendering comparison with surgical series difficult (e.g., $[6,21-26])$. The intend of this analysis therefore was to focus on metastatic CRC patients only, to avoid bias by histology and to investigate the effect of LC on OS after SBRT for lung or liver metastases. In addition, we wanted to investigate the effect of metastatic site (liver, lung) on tumor control rates, as there have been

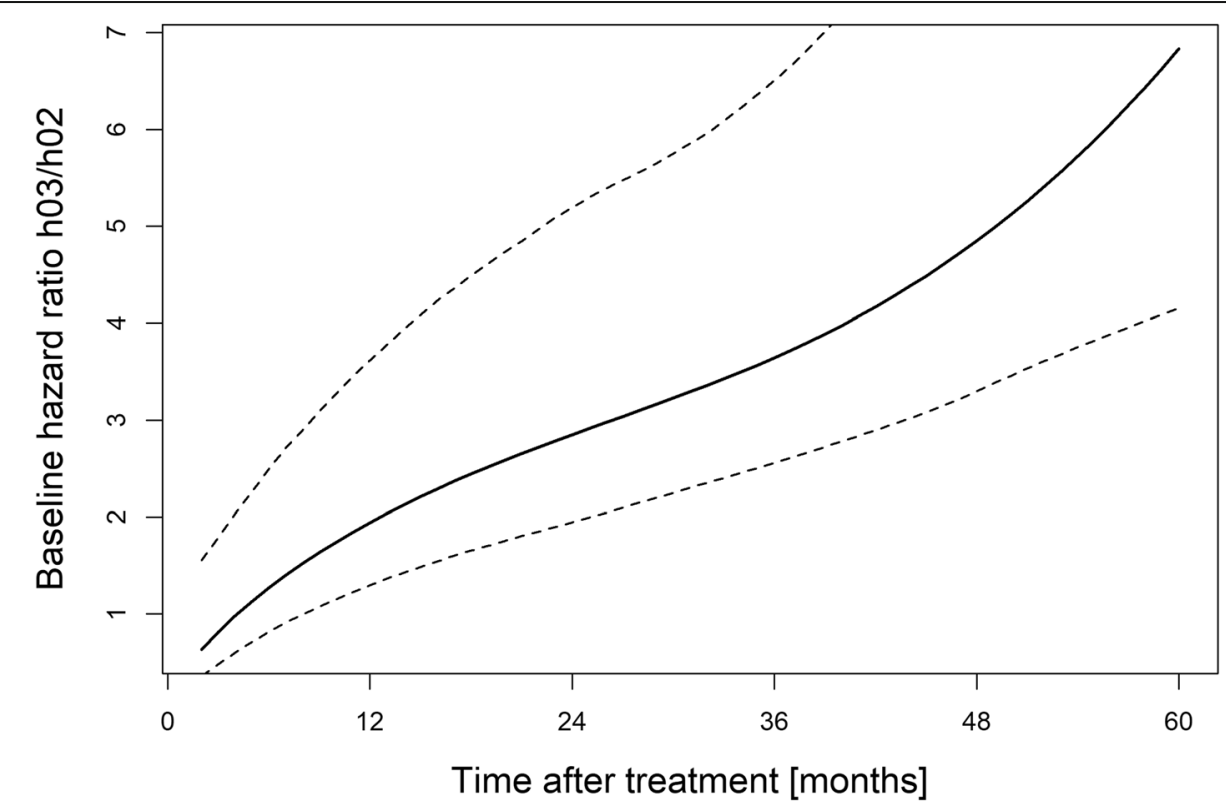

Fig. 3 Baseline hazard ratio between transitions 3 and 2 as a function of follow-up time after treatment. Ratios greater than 1 indicate a greater risk of death if a patient has experienced a local recurrence prior to the time considered. The dashed lines indicate the $95 \%$ confidence band based on 500 Monte Carlo simulations of the baseline hazards. A very similar trend is observed when computing the baseline hazard ratio for a lung metastasis patient (coded with tumor site $=1$ ), although the confidence bands are wider (not shown) 

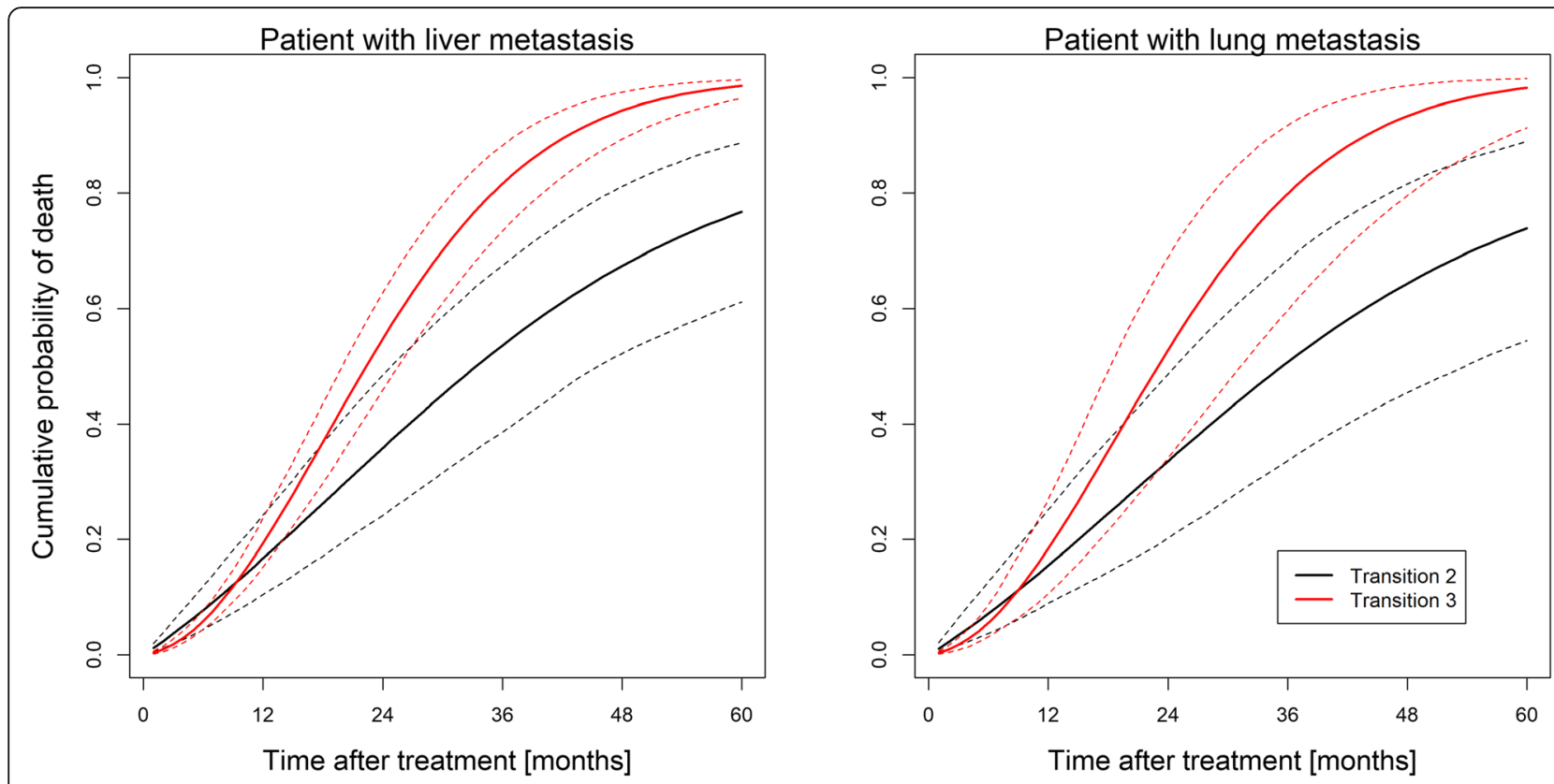

Fig. 4 Cumulative probability of making transitions 2 (black) and 3 (red) as a function of follow-up time after treatment. Predictions are for an average patient (male, KPS $\geq 90$, age < 66 years, one metastasis, given chemotherapy) with a liver (left panel) or lung (right panel) metastasis, respectively. 95\% confidence bands based on 500 Monte Carlo samples are shown as dotted lines. All predictions are averaged over different imputations of the chemotherapy covariate. Note that after some short initial time the probability of transition 3 starts to exceed that of transition 2, indicating a higher probability of death if the metastasis has not been controlled

observations that site-specific differences in the response of CRC metastases to ionizing radiation might exist [27-33].

Our analysis is the first to specifically address the question whether the site of colorectal cancer metastases plays a role in the response to SBRT treatment, but also and even more importantly whether LC also translates into improved OS. Applying an illness-death type multistate model to a total of 500 SBRT treatments of CRC metastases, we studied differences between lung and liver metastases with respect to LC and OS and the interplay between both outcomes. The structure of the illness-death model naturally accounts for the relation between LC and OS which is not the case if LC would be treated as a time-dependent covariate.

\section{Dose-response analysis and factors influencing local control}

For achieving LC, we identified radiation dose or, more precisely, $\mathrm{BED}_{\mathrm{iso}}$ as the most important variable. However, the dose-response relationship was strongly influenced by tumor site and whether chemotherapy had been given prior to SBRT. The results are also in line with our previous modeling studies of the full liver and lung metastases cohorts from which the data analyzed here had been extracted: Without taking any confounding factors into account, the BED needed to achieve 90\% TCP after 1 year was estimated as $192 \mathrm{~Gy}_{10}\left(\mathrm{SE} 37 \mathrm{~Gy}_{10}\right.$ ) for liver metastases [11], but $110 \mathrm{~Gy}_{10}\left(\mathrm{SE} 17 \mathrm{~Gy}_{10}\right)$ for lung metastases [34]. Further data providing evidence for an influence of tumor site on LC have been published by Ahmed et al. [5] who reported 100\% LC rates at 2-years after SBRT treatment of CRC lung metastases, but only $73.0 \%$ in liver metastases of similar sizes using the same prescription of $60 \mathrm{~Gy}$ in 5 fractions. Although their number of 29 lesions in total was small, these differences were statistically significant $(p=0.026)$ and attributed to differences in the parenchyma and vasculature of liver and lung tissue [5]. A significantly larger $\alpha /$ $\beta$ ratio for hepatic compared to lung metastases, as recently estimated in a meta-regression study by Klement [35], would be consistent with such an explanation. In contrast to the assumption of different site dependent radiation sensitivity by Ahmed et al., another interpretation for the difference in LC between lung and liver metastases could be the challenges in target volume delineation, image guided radiation delivery and motion management technique. While this is supported by a recent dose-response analysis of our large SBRT database of lung and liver metastases, indicating that the motion management technique significantly impacts LC for liver, but not lung metastases ([11] and unpublished data), the significance of tumor site persisted even after controlling for motion management in our analysis. One limitation of this analysis is that information on the within-organ location of individual metastases was 


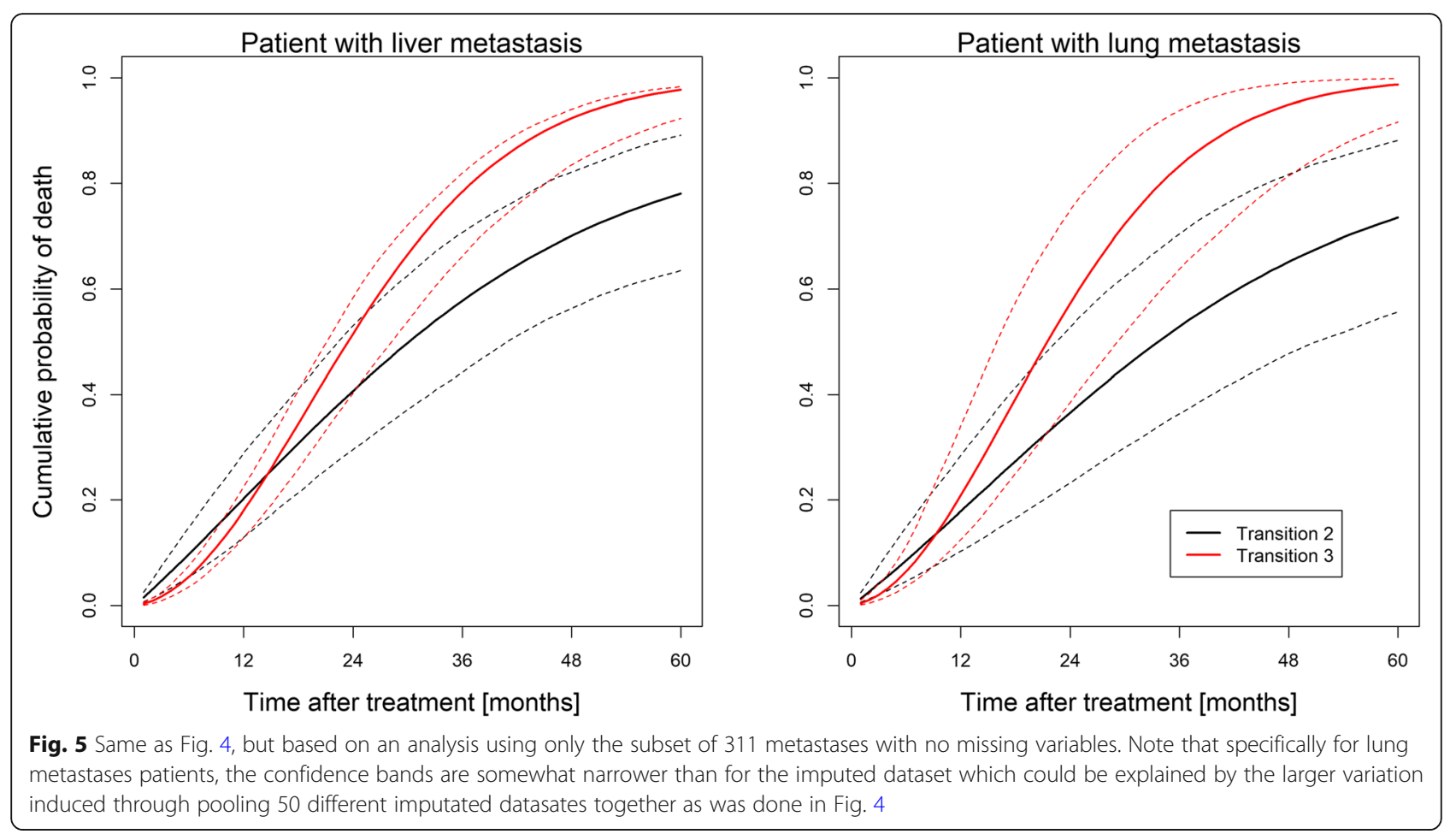

lacking; in the full lung metastases data set peripheral tumor location (compared to central location) was associated with improved TCP [34].

Our finding of chemotherapy prior to SBRT being a negative predictive factor for LC is certainly intriguing and needs further investigation. However, we believe that this finding may explain the frequent notion that CRC metastases (which are frequently heavily pre-treated) are more radioresistant compared to other histologies [11]. Unfortunately, detailed information on the type of chemotherapy was lacking, but in particular adjuvant oxaliplatin (FOLFOX) therapy has been shown to be associated with worse outcomes in patients who later developed liver metastases, consistent with the increased accumulation of new somatic mutations and acquirement of therapy resistance [36]. Another explanation might be that patients having received chemotherapy prior to SBRT would be more likely to have node-positive primaries, indicative of more aggressive tumors.

\section{The impact of local control on overall survival}

The dependence of OS on LC is the most relevant finding of this study. For the first time reported in SBRT treated metastatic patients, a survival benefit was observed, if LC of individual metastases has been achieved. Our results are consistent with surgery outcomes showing significantly longer 5-year OS after R0 compared to R1 resection of hepatic metastases in CRC patients with or without prior chemotherapy [22].
As Figs. 3 and 4 show, the probability of dying after a local relapse soon exceeds that of dying if no relapse has occurred. However, this difference becomes apparent only after about 10 months of follow-up (separation of the transition curves in Fig. 4), implying that LC impacts OS if the projected prognosis is beyond this time point. Conversely, if the projected survival is less than 10 months, achieving LC is clinically not relevant.

This finding directly relates to two important aspects to achieve a possible survival benefit: patient and radiation dose selection.

Selection of an appropriate radiation dose is directly related to a previous analysis by our group developing a model of TCP depending on both dose and time [34]. In this model, the dose needed for a certain probability of $\mathrm{LC}$ was time dependent: the longer the time interval the higher the respective BED necessary for the same TCP. Translated to our current findings this implies that if the projected prognosis is beyond 12 months, BED needs to be high enough in order to assure long-term LC and improved OS. Therefore, beyond an organs-at-risk adapted dose selection, a time-adjusted BED with clinically relevant LC (e.g. 90\% TCP at 2 years) should be employed in metastasized CRC patients with presumed longer-term survival. Examples of such dose prescriptions based on our model are given in Table 3. The high doses predicted by our model as necessary to achieve high long-term TCP in chemotherapy pre-treated metastases may be very difficult to achievable clinically due to normal tissue dose constraints, especially in the liver. However, besides 
the fact that these doses are not necessarily generalizable to other target populations, they primarily highlight the complicated interplay between patient prognosis and dose escalation.

That the results of this retrospective study are specific to our cohort and not necessarily generalizable to a different target population poses a major limitation for the interpretation of our results. While similar analyses in different cohorts and future prospective trials need to be conducted, specifically the causal claim that LC influences OS would be further supported by studying potential underlying mechanisms [37]. Another limitation is that our cohort lacked detailed information on the pre-SBRT treatment history of individual patients, in particular concerning types of chemotherapy and local therapies such as previous liver or lung resections. It follows that patients probably had varying prognoses of both LC and OS at the time of SBRT depending on factors that we were not able to account for in our model. A prospective study with a more balanced patient cohort, possibly as a randomized trial applying different fractionation schedules, would be very helpful for confirming our finding that achieving long-term LC has a positive impact on OS. Finally, a large number of metastases had at least one missing variable (189 or $37.8 \%$ of the sample) which we decided to impute and which in principal could have influenced our results. However, re-analyzing the sample of 311 metastases with complete entries, we obtained qualitatively similar results to the main analysis, in particular regarding the importance of predictors of transitions $1-3$, and the impact of LC on OS (Fig. 5).

Current patient selection criteria for local ablative treatment are based on surgical analyses of oligometastatic CRC patients with liver and lung metastases and relate clinical parameters with prognosis. In optimally selected candidates OS rates of up to $40 \%$ at 5 years can be achieved for those patients. Conversely, even patients who only fulfill a subset of these criteria may benefit from local intervention. Therefore, a finer grained approach would be desirable to estimate an individual's projected survival based on a combination of clinical parameters to guide the optimal decision regarding local ablative treatment. To this end, we recently developed a nomogram for oligometastatic lung disease treated with SBRT based on clinical parameters: KPS, type of the primary tumor, control of the primary tumor, maximum diameter of the largest treated metastasis and number of metastases (1 versus >1) [38]. These parameters are readily available and the nomogram allows deriving 4 prognostic groups which could serve as guidance for patient selection regarding local ablative treatment of oligometastatic disease. Still, validation of this nomogram in other metastatic disease sites is warranted to generalize its applicability in oligometastatic disease.

\section{Conclusion}

Utilizing a large patient cohort from a multi-institutional database and focusing on CRC patients with lung or liver metastases, the variables radiation dose, tumor site and pre-SBRT chemotherapy could be identified as predictive factors for LC after SBRT. Most importantly, application of an illness-death model revealed an association between achieving LC with SBRT and OS: patients without local recurrence had a significantly lower risk of death when adjusting for other influencing factors such as tumor site (lung vs. liver), maximum tumor volume or performance status. However, this was only observed in patients with a projected OS estimate of approximately 12 months or more, underlining the importance of proper patient selection for dose-intensified SBRT.

\section{Abbreviations \\ BED: Biologically effective dose; Cl: Confidence interval; CRC: Colorectal cancer; DEGRO: German Society for Radiation Oncology; GTV: Gross tumor volume; KPS: Karnofsky performance score; LC: Local control; NSCLC: Non small cell lung cancer; OS: Overall survival; PTV: Planning target volume; SBRT: Stereotactic Body Radiation Therapy; SE: Standard error; TCP: Tumor control probability}

\section{Acknowledgments}

Part of this work has been presented in a talk at the 23rd annual meeting of the German Society for Radiation Oncology (DEGRO) in Berlin and published as an abstract (Klement et al., Strahlenther Onkol (2017) (suppl) 193:S32-S33. https://link.springer.com/content/pdf/10.1007\%2Fs00066-017-1137-6.pdf).

\section{Funding}

None.

\section{Availability of data and materials}

The data that support the findings of this study are available from the AG Stereotaxie und Radiochirurgie of the German Society of Radiation Oncology, but restrictions apply to the availability of these data, which were used under license for the current study, and so are not publicly available. Data are however available from the authors upon reasonable request and with permission of AG Stereotaxie und Radiochirurgie of the German Society of Radiation Oncology.

\section{Authors' contributions}

RJK, MG and NA drafted the manuscript, analyzed and interpreted the data. RJK and NA performed and approved the statistical analysis. RJK, NAS, SA HA, MA, GB, OB, JBH, TB, MD, MJE, IE, SG, DH, PH, CH, GH, DI, HK, NDK, RK, VL, $F L, C O, A P, C P, J R, T S, E S, R S, S W, A W, M G, N A$ took part in the data collection, interpretation procedure, drafting and reviewing of the article. All authors approved the presented article before submission and take responsibility for the integrity of the analysis and the respective manuscript.

\section{Ethics approval and consent to participate}

The multicenter data collection and analysis was approved by the Ethics committee of the Kanton Zurich, Switzerland (BASEC-Nr. 2016-00744) and in addition to local regulations also covered the following institutions:

1. University Hospital Zürich, Department of Radiation Oncology, University of Zurich, Zurich, Switzerland.

2. Strahlentherapie Bautzen, Department of Radiation Oncology, Bautzen, Germany

3. University of Munich - LMU Munich, Department of Radiation Oncology,Munich, German 
4. University Hospital Basel, Department of Radiation Oncology, Basel, Switzerland

5. University Medical Center Hamburg-Eppendorf, Department of Radiation Oncology, Hamburg, Germany

6. Strahlenzentrum Hamburg, Department of Radiation Oncology, Hamburg, Germany

7. University Hospital of Cologne, Department of Radiation Oncology, Cologne, Germany

8. University Hospital Würzburg, Department of Radiation Oncology, Würzburg, Germany

9. University Hospital Halle, Department of Radiation Oncology, Halle, Germany

10. Klinikum Passau, Radiation Oncology, Passau, Germany

If necessary, the data collection of the individual participating centers was approved according to local regulations and approved by the respective local ethics committees. The following ethics committees and regulatory bodies were involved in this local approval process:

1. Medizinische Ethik-Komission II, Medizinische Fakultät Mannheim; 2014-413 M-MA-§23bMPG: University Hospital Mannheim, Department of Radiation Oncology, University of Heidelberg, Mannheim, Germany.

2. Ethikkommission der Medizinischen Fakultät Heidelberg: S459-2010:

3. Ethikkommission der Medizinischen Fakultät der Technischen Universität München; 84/16S: Klinikum rechts der Isar- Technische Universität München, Department of Radiation Oncology, Munich, Germany

4. Ethikkommission an der Medizinischen Fakultät der Universität Rostock, A2016-0008:

a. Universitätsklinikum Schleswig-Holstein, Department of Radiation Oncology, Kiel/Lübeck, Germany.

b. University Hospital Rostock, Department of Radiation Oncology, Rostock, Germany.

5. Ethikkommission der Universität Freiburg, 462/12: University Hospital Freiburg, Department of Radiation Oncology, Freiburg, Germany

6. Ärztekammer: Bezirksärztekammer Nord-Württemberg, Jahnstr. 5, 70,597 Stuttgart: RadioChirurgicum CyberKnife Südwest, Radiation Oncology, Göppingen, Germany.

7. Ethikkommission der Bayerischen Ärztekammer, mb BO 16002: Krankenhaus Barmherzige Brüder, Department of Radiation Oncology, Regensburg, Germany

The participants consent was written as part of the main ethics approval.

\section{Consent for publication}

Not applicable.

\section{Competing interests}

Marciana Duma and Christian Ostheimer are members of the editorial board (Associate editors) of BMC Cancer. NA confirms that all other authors have nothing to declare at the time of submission and that there are no competing interests to declare.

\section{Publisher's Note}

Springer Nature remains neutral with regard to jurisdictional claims in published maps and institutional affiliations.

\section{Author details}

${ }^{1}$ Department of Radiation Oncology, Leopoldina Hospital Schweinfurt, Schweinfurt, Germany. ${ }^{2}$ Department of Radiation Oncology, University Hospital Jena, Jena, Germany. ${ }^{3}$ Department of Radiation Oncology, University Hospital Freiburg, Freiburg, Germany. ${ }^{4}$ Strahlentherapie Bautzen, Bautzen, Germany. 'Department of Radiation Oncology, Hospital Barmherzige Brueder, Regensburg, Germany. ${ }^{6}$ RadioChirurgicum CyberKnife Suedwest, Goeppingen, Germany. ${ }^{7}$ Department of Radiation Oncology Universitaetsklinikum Schleswig-Holstein, Luebeck, Germany. ${ }^{8}$ Department of Radiation Oncology, University Medical Center Mannheim, University of Heidelberg, Mannheim, Germany. ${ }^{9}$ Department of Radiation Oncology, Klinikum rechts der Isar- Technische Universitaet Muenchen, Munich,
Germany. ${ }^{10}$ Department of Radiation Oncology, University Hospital Aachen, Aachen, Germany. ${ }^{11}$ Department of Radiation Oncology, University Hospital Muenster, Muenster, Germany. ${ }^{12}$ Department of Radiation Oncology, Ludwig Maximilians University Munich, Munich, Germany. ${ }^{13}$ Department of Radiation Oncology, University Hospital Heidelberg, Heidelberg, Germany.

${ }^{14}$ Department of Radiation Oncology, University Hospital Magdeburg,

Magdeburg, Germany. ${ }^{15}$ Department of Radiotherapy and Special Oncology, Medical School Hannover, Hanover, Germany. ${ }^{16}$ Department of Radiation Oncology, University of Rostock, Rostock, Germany. ${ }^{17}$ Department of Radiation Oncology, University Hospital Frankfurt, Frankfurt, Germany. ${ }^{18}$ Department of Radiation Oncology, Hospital Augsburg, Augsburg, Germany. ${ }^{19}$ Department of Radiation Oncology, University Hospital Bern, Bern, Switzerland. ${ }^{20}$ Department of Radiation Oncology, Helios Klinikum Berlin Buch, Berlin, Germany. ${ }^{21}$ Department of Radiation Oncology, University Hospital Wuerzburg, Wuerzburg, Germany. ${ }^{22}$ Department of Radiation Oncology, Faculty of Medicine and University Hospital Carl Gustav Carus, Technische Universität Dresden, Dresden, Germany. ${ }^{23}$ Department of Radiation Oncology, University Hospital Halle, Halle, Germany. ${ }^{24}$ Department of Radiation Oncology, University Hospital Hamburg, Hamburg, Germany.

${ }^{25}$ Department of Radiation Oncology, University Hospital Basel, Basel,

Switzerland. ${ }^{26}$ Strahlenzentrum Hamburg, Hamburg, Germany. ${ }^{27}$ Department of Radiation Oncology, Hospital Heidenheim, Heidenheim, Germany. ${ }^{28}$ Department of Radiation Oncology, University Hospital of Cologne, Cologne, Germany. ${ }^{29}$ Department of Radiation Oncology, Klinikum Passau, Passau, Germany. ${ }^{30}$ Department of Radiotherapy and Radiation Oncology, Philipps-University Marburg, University Hospital Giessen and Marburg, Marburg, Germany. ${ }^{31}$ Department of Radiation Oncology, University Hospital Zurich, University of Zurich, Rämistrasse 100, 8091 Zurich, Switzerland.

Received: 10 June 2018 Accepted: 11 February 2019

Published online: 26 February 2019

\section{References}

1. Arnold M, Sierra MS, Laversanne M, Soerjomataram I, Jemal A, Bray F. Global patterns and trends in colorectal cancer incidence and mortality. Gut. 2017 ; 66(4):683-91. https://doi.org/10.1136/gutjnl-2015-310912.

2. Patanaphan V, Salazar OM. Colorectal cancer: metastatic patterns and prognosis. South Med J. 1993;86:38-41.

3. Fong $Y$, Fortner J, Sun RL, Brennan MF, Blumgart LH. Clinical score for predicting recurrence after hepatic resection for metastatic colorectal cancer: analysis of 1001 consecutive cases. Ann Surg. 1999:230:309-18 discussion 318-21.

4. Casiraghi M, De PT, Brambilla D, Ciprandi B, Galetta D, Borri A, et al. A 10-year single-center experience on 708 lung metastasectomies: the evidence of the "international registry of lung metastases.". J Thorac Oncol. 2011;6:1373-8.

5. Ahmed KA, Fulp WJ, Berglund AE, Hoffe SE, Dilling TJ, Eschrich SA, et al. Differences between Colon Cancer primaries and metastases using a molecular assay for tumor radiation sensitivity suggest implications for potential Oligometastatic SBRT patient selection. Int J Radiat Oncol Biol Phys. 2015;92:837-42.

6. MacDermed DM, Weichselbaum RR, Salama JK. A rationale for the targeted treatment of Oligometastases with radiotherapy. J Surg Oncol. 2008:98:202-6.

7. Salama J, Hasselle M, Chmura S. Stereotactic body radiotherapy for multisite extracranial oligometastases. Cancer. 2012;118:2962-70.

8. Gomez DR, Blumenschein GR, Lee JJ, Hernandez M, Ye R, Camidge $D R$, et al. Local consolidative therapy versus maintenance therapy or observation for patients with oligometastatic non-small-cell lung cancer without progression after first-line systemic therapy: a multicentre, randomised, controlled, phase 2 study. Lancet Oncol. 2016;0:578-83.

9. Ruers T, Van Coevorden F, Punt CJA, Pierie J-PEN, Borel-Rinkes I, Ledermann JA, et al. Local treatment of unresectable colorectal liver metastases: Results of a randomized phase II trial. J Natl Cancer Inst. 2017;109(9).

10. Rieber J, Streblow J, Uhlmann L, Flentje M, Duma M, Ernst I, et al. Stereotactic body radiotherapy (SBRT) for medically inoperable lung metastases - a pooled analysis of the German working group "stereotactic radiotherapy". Lung Cancer. 2016;97:51-8. 
11. Klement RJ, Guckenberger M, Alheid H, Allgäuer M, Becker G, Blanck O, et al. Stereotactic body radiotherapy for oligo-metastatic liver disease - influence of pre-treatment chemotherapy and histology on local tumor control. Radiother Oncol. 2017;123:227-33.

12. Rondeau V, Pignon J-P, Michiels S. A joint model for the dependence between clustered times to tumour progression and deaths: a metaanalysis of chemotherapy in head and neck cancer. Stat Methods Med Res. 2011:32:5380.

13. Haneuse S, Lee KH. Semi-competing risks data analysis. Circ Cardiovasc Qual Outcomes. 2016;9:322-31.

14. Duchateau $L$, Janssen $P$. The frailty model. 1st ed. New York: Springer science+business Media; 2008.

15. Xu J, Kalbfleisch JD, Tai B. Statistical analysis of illness-death processes and semicompeting risks data. Biometrics. 2010;66:716-25.

16. Rondeau V, Mazroui Y, Gonzalez JR. Frailtypack: an R package for the analysis of correlated survival data with frailty models using penalized likelihood estimation or parametrical estimation. J Stat Softw. 2012;47:1-28.

17. Harrell FE Jr. Regression Modeling Strategies. 2nd ed. New York: Springer; 2015.

18. Van Buuren S, Groothuis-Oudshoorn K. Mice: multivariate imputation by chained equations in R. J Stat Softw. 2011;45:1-67.

19. Gelman A. Scaling regression inputs by dividing by two standard deviations. Stat Med. 2008:27:2865-73.

20. Hellman S, Weichselbaum RR. Oligometastases. J Clin Oncol. 1995;13:8-10.

21. Milano MT, Katz AW, Zhang H, Okunieff P. Oligometastases treated with stereotactic body radiotherapy: long-term follow-up of prospective study. Int J Radiat Oncol Biol Phys. 2012;83:878-86.

22. Angelsen J-H, Horn A, Eide GE, Viste A. Surgery for colorectal liver metastases: the impact of resection margins on recurrence and overall survival. World J Surg Oncol. 2014;12:127.

23. Okunieff P, Petersen AL, Philip A, Milano MT, Katz AW, Boros L, et al. Stereotactic body radiation therapy (SBRT) for lung metastases. Acta Oncol. 2006;45:808-17.

24. Katz AW, Carey-Sampson M, Muhs AG, Milano MT, Schell MC, Okunieff P. Hypofractionated stereotactic body radiation therapy (SBRT) for limited hepatic metastases. Int J Radiat Oncol Biol Phys. 2007;67:793-8.

25. Rusthoven KE, Kavanagh BD, Burri SH, Chen C, Cardenes H, M a C, et al. Multi-institutional phase I/II trial of stereotactic body radiation therapy for lung metastases. J Clin Oncol. 2009;27:1579-84.

26. Rusthoven KE, Kavanagh BD, Cardenes H, Stieber WW, Burri SH, Feigenberg SJ, et al. Multi-institutional phase I/II trial of stereotactic body radiation therapy for liver metastases. J Clin Oncol. 2009;27:1572-8.

27. Wulf J, Guckenberger M, Haedinger U, Oppitz U, Mueller G, Baier K, et al. Stereotactic radiotherapy of primary liver cancer and hepatic metastases. Acta Oncol (Madr). 2006;45:838-47.

28. Stintzing S, Hoffmann R-T, Heinemann V, Kufeld M, Rentsch M, Muacevic A. Radiosurgery of liver tumors: value of robotic radiosurgical device to treat liver tumors. Ann Surg Oncol. 2010;17:2877-83.

29. Takeda A, Kunieda E, Ohashi T, Aoki Y, Koike N, Takeda T. Stereotactic body radiotherapy (SBRT) for oligometastatic lung tumors from colorectal cancer and other primary cancers in comparison with primary lung cancer. Radiother Oncol. 2011;101:255-9.

30. Singh D, Chen Y, Hare MZ, Usuki KY, Zhang H, Lundquist T, et al. Local control rates with five-fraction stereotactic body radiotherapy for oligometastatic cancer to the lung. J Thorac Dis. 2014;6:369-74.

31. Thibault I, Poon I, Yeung L, Erler D, Kim A, Keller B, et al. Predictive factors for local control in primary and metastatic lung tumours after four to five fraction stereotactic ablative body radiotherapy: a single institution's comprehensive experience. Clin Oncol (R Coll Radiol). 2014;26:713-9.

32. Andratschke N, Parys A, Stadtfeld S, Wurster S, Huttenlocher S, Imhoff D, et al. Clinical results of mean GTV dose optimized robotic guided SBRT for liver metastases. Radiat Oncol. 2016;11:74.

33. Ahmed KA, Caudell JJ, El-Haddad G, Berglund AE, Welsh EA, Yue B, et al. Radiosensitivity differences between liver metastases based on primary histology suggest implications for clinical outcomes after stereotactic body radiation therapy. Int J Radiat Oncol Biol Phys. 2016; 95:1399-404.

34. Klement RJ, Allgäuer M, Andratschke N, Blanck O, Boda-Heggemann J, Dieckmann K, et al. Bayesian cure rate modeling of local tumor control: evaluation in stereotactic body radiotherapy for pulmonary metastases. Int J Radiat Oncol. 2016;94:841-9.
35. Klement RJ. Radiobiological parameters of liver and lung metastases derived from tumor control data of 3719 metastases. Radiother Oncol. 2017;123: 218-26.

36. Andreou A, Kopetz S, Maru DM, Chen SS, Zimmitti G, Brouquet A, et al. Adjuvant chemotherapy with FOLFOX for primary colorectal Cancer is associated with increased somatic gene mutations and inferior survival in patients undergoing hepatectomy for Metachronous liver metastases. Ann Surg. 2012;256:642-50.

37. Russo F, Williamson J. Interpreting causality in the health sciences. Int Stud Philos Sci. 2007;21:157-70.

38. Tanadini-Lang S, Rieber J, Filippi AR, Fode MM, Streblow J, Adebahr S, et al. Nomogram based overall survival prediction in stereotactic body radiotherapy for oligo-metastatic lung disease. Radiother Oncol. 2017;123: $182-8$.

\section{Ready to submit your research? Choose BMC and benefit from:}

- fast, convenient online submission

- thorough peer review by experienced researchers in your field

- rapid publication on acceptance

- support for research data, including large and complex data types

- gold Open Access which fosters wider collaboration and increased citations

- maximum visibility for your research: over $100 \mathrm{M}$ website views per year

At BMC, research is always in progress.

Learn more biomedcentral.com/submissions 